\title{
Evaluation of uterine scar healing by transvaginal ultrasound in 607 nonpregnant women with a history of cesarean section
}

\author{
Xingchen Zhou ${ }^{1 \dagger}$, Tao Zhang $^{2 \dagger}$, Huayuan Qiao ${ }^{3}$, Yi Zhang ${ }^{1}$ and Xipeng Wang ${ }^{1^{*}}$ (1)
}

\begin{abstract}
Background: Caesarean scar defect (CSD) seriously affects female reproductive health. In this study, we aim to evaluate uterine scar healing by transvaginal ultrasound (TVS) in nonpregnant women with cesarean section (CS) history and to build a predictive model for cesarean scar defects is very necessary.

Methods: A total of 607 nonpregnant women with previous CS who have transvaginal ultrasound measurements of the thickness of the lower uterine segment. The related clinical data were recorded and analyzed.

Results: All patients were divided into two groups according to their clinical symptoms: Group $A(N=405)$ who had no cesarean scar symptoms, and Group B ( $N=141)$ who had cesarean scar symptoms. The difference in frequency of CS, uterine position, detection rate of CSD and the residual muscular layer (TRM) of the CSD were statistically significant between groups; the TRM measurements of the two groups were $(\mathrm{mm}) 5.39 \pm 3.34$ versus $3.22 \pm 2.33, P<0.05$. All patients were divided into two groups according to whether they had CSDs: Group C ( $N=337)$ who had no CSDs, Group D ( $N=209)$ who had CSDs on ultrasound examination. The differences in frequency of CS, uterine position, TRM between groups were statistically significant $(P<0.05)$. In the model predicting CSDs by TRM with TVS, the area under the ROC curve was 0.771 , the cut-off value was $4.15 \mathrm{~mm}$. The sensitivity and specificity were $87.8 \%$ and $71.3 \%$, respectively.
\end{abstract}

Conclusions: Patients with no clinical symptoms had a mean TRM on transvaginal ultrasonography of $5.39 \pm 3.34 \mathrm{~mm}$, which could be used as a good reference to predict the recovery of patients with CSDs after repair surgery.

Keywords: Cesarean section, Cesarean scar defect (CSD), Thickness of residual myometrial (TRM), Transvaginal ultrasound (TVS), Predictive model

\section{Background}

With the increasing cesarean section (CS) rate, a growing number of studies suggest that the occurrences of longterm consequences of $\mathrm{CS}$ are related to the incomplete

\footnotetext{
*Correspondence: wangxipeng@xinhuamed.com.cn

${ }^{+}$Xingchen Zhou and Tao Zhang contributed equally to this work

${ }^{1}$ Department of Obstetrics and Gynecology, Xinhua Hospital,

Affiliated to Shanghai Jiaotong University, No. 1665 Kong Jiang Road,

Shanghai 200092, China

Full list of author information is available at the end of the article
}

healing of the CS scar in the uterus, which leads to the development of cesarean scar defects (CSDs) $[1,2]$. In routine ultrasound examinations of the uteri of nonpregnant women with a history of at least one CS, the prevalence of CS scar defects ranges from 24 to $70 \%$ [3]. Some patients with CSDs are asymptomatic, but several investigators have reported an association between these defects and abnormal bleeding and postmenstrual spotting $[4,5]$; CSDs can also cause chronic pelvic pain and even infertility [6]. Moreover, the development of CSDs 
seems to be on the rise, and CSDs can occur on a spectrum of disorders starting with cesarean scar (CS) ectopic pregnancy [7], to increased incidence of placenta previa and uterine rupture associated with major maternal morbidity, and even mortality [8]; this is a very important medical problem that affects a large population of women. Therefore, the detection and diagnosis of uterine incision diverticulum is very important, especially in asymptomatic patients.

In particular, the thickness of the residual muscular layer (TRM) of the CSD directly affects the severity of the clinical symptoms and the risk of maternal complications, such as uterine rupture in subsequent pregnancy [8]. Therefore, it is very important to evaluate uterine scar healing in nonpregnant women with previous cesarean section. Transvaginal ultrasound is the most convenient and noninvasive means of examination. However, most of the studies about uterine incision healing included measurements of the lower uterine segment (LUS) thickness in pregnant women with a previous caesarean delivery $[9,10]$. There is a lack of transvaginal ultrasound evaluations and multisampling statistical analyses for LUS measurements in nonpregnant women. There is no clinical reference standard to evaluate the efficacy of surgical repair in CSD patients. Therefore, this study investigated the ability of transvaginal ultrasound to detect cesarean scars and the prevalence of scar defects in nonpregnant subjects. By measuring the thickness of the scar in the lower segment of the uterus, diameters of the CSDs, uterine position and other imaging data, the range of TRM values in nonpregnant women with a CS history could be determined. The purpose of this study was to determine the value of LUS thickness that predicts good healing of the uterine incision after cesarean section and to reveal the clinical indicators that predict CSD and could be used clinically in patient management.

\section{Methods}

\section{Patients selection}

In this prospective study, 607 patients who presented at the Shanghai First Maternity and Infant Hospital, Tongji University from October 2016 to October 2018 were enrolled. Inclusion criteria: All patients had undergone at least one $\mathrm{C}$-section and were currently not pregnant with or without a prolonged menstruation period. Exclusion criteria: (1) Patients had a history of other uterine surgery that could have changed their cavity anatomy. (2) The patient had a congenital uterine abnormality, such as a biangular uterus. (3) The patient had undergone classical cesarean section. All subjects underwent transvaginal ultrasonography (TVU) to assess uterine scar healing. This study was approved by the Ethics Committee of Shanghai First Maternity and Infant Hospital, affiliated with Tongji University (KS1512), and was conducted in accordance with the Declaration of Helsinki. All patients enrolled in the study signed informed consent forms.

\section{Methods}

Each subject underwent a transvaginal ultrasound of the cervix, uterus, and adnexa, which was performed by one experienced sonographer blinded to the woman's history. Ultrasound examinations were performed using a color ultrasonic diagnostic apparatus, Philips iU22, Philips HD15, GE E8. Both ultrasound devices were equipped with a 4-9 $\mathrm{MHz}$ transvaginal probe. Cesarean scar defects were recorded as either present or absent. If a CSD was present, the defect was measured in two dimensions in the sagittal plane (anterior-posterior and cephalad-caudal) and transversely in the coronal plane. Three different values of length, width, and depth as well as TRM were taken, and the mean value of these parameters was considered the actual data. The frequency of scar identification, as well as the presence of fluid within the scar ("scar defect"), was recorded and later compared with the self-reported obstetric history. Photo documentation was recorded with the presence or absence of a cesarean scar defect and the presence or absence of fluid within the scar (Fig. 1). Additional images of the endometrial stripe thickness, presence or absence of polyps or myomas, and ovarian volumes were also recorded. Each subject was assigned a study number at enrollment. Data obtained from the questionnaires (including age, contact information, number of cesarean sections, time of last cesarean section, menstruation and so on) and from the ultrasound were entered separately.

\section{Statistical analysis}

Measurement data, such as length, width and depth as well as the TRM of the bottom of the CSD were expressed as the mean $\pm \mathrm{SD}$. Continuous variables were compared by paired $\mathrm{t}$-tests. The results of them were used to trace out receiver operating characteristic (ROC) curves to predict CSDs. With the use of these curves, the threshold values of each variable were set. Variables that achieved statistical significance in the univariate analysis were subsequently included in the multivariate analysis. A $P<0.05$ (two-tailed) was considered statistically significant. SPSS version 24.0 was used for all statistical calculations.

\section{Results}

\section{Patient characteristics}

A total of 607 consenting women with a history of at least one cesarean section were enrolled in the study over the period from October 2016 to October 2018; 17 subjects who did not undergo transvaginal ultrasound and 43 patients who underwent transvaginal ultrasound 

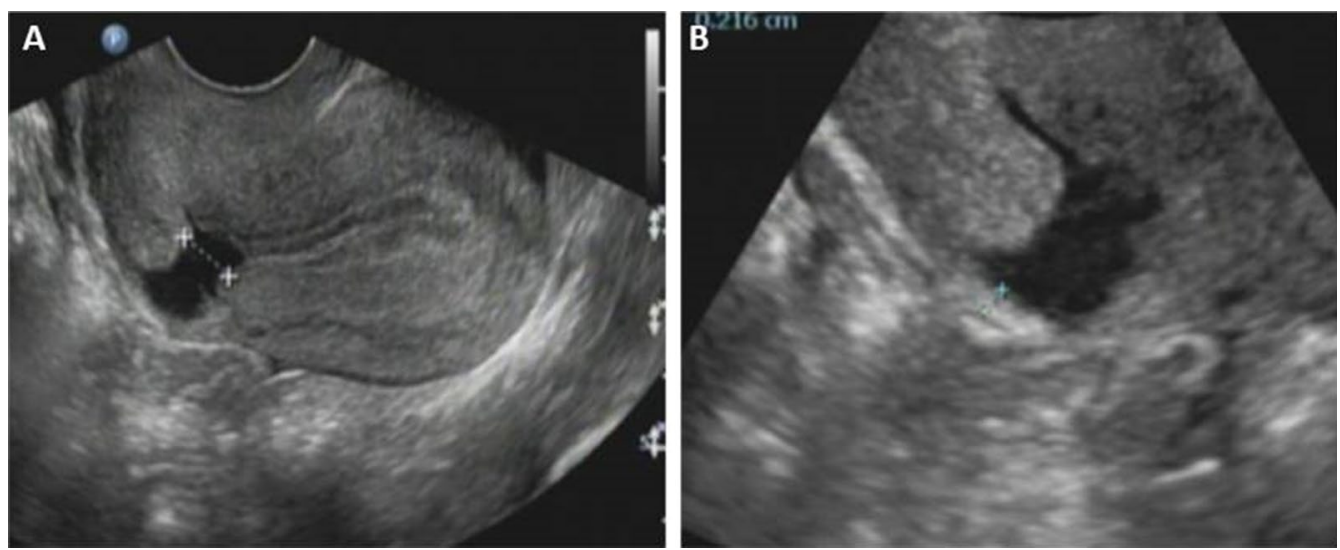

Fig. 1 a CSD under transvaginal ultrasound; b TRM under transvaginal ultrasound

Table 1 Patient characteristics

\begin{tabular}{llll}
\hline Group & noCSD & CSD & Total \\
\hline asymptomatic & 310 & 95 & 405 \\
symptomatic & 27 & 114 & 141 \\
Total & 337 & 209 & 546 \\
\hline
\end{tabular}

but did not have TRM measurements were excluded. A total of 546 women completed the study. All patients were divided into two groups according to their clinical symptoms: Group A ( $\mathrm{N}=405)$ included women who had no cesarean scar symptoms, and Group B $(\mathrm{N}=141)$ included women who had cesarean scar symptoms, such as postmenstrual spotting, prolonged menstruation, and continuous brown discharge. The women were also divided into two groups according to their CSD status: Group $\mathrm{C}(\mathrm{N}=337)$ included women who had no CSDs on the ultrasound examination, and Group D $(\mathrm{N}=209)$ included women who had CSDs on the ultrasound examination. The baseline characteristics of the 546 patients are presented in Table 1 .

\section{Clinical characteristics of the patients in Group A and Group B}

Group A $(\mathrm{N}=405)$ included women who had no clinical symptoms. Group B $(\mathrm{N}=141)$ included women who had cesarean scar symptoms, such as postmenstrual spotting, prolonged menstruation, and continuous brown discharge. The clinical data and uterine incision healing data were compared between the two groups. The results showed that the average age of the two groups of patients was $35.09 \pm 5.32$ versus $34.00 \pm 4.83$ years old, and the median age of the two groups was 34 years old. The number of women who
Table 2 Characteristics of clinical data between symptomatic group (Group A) and asymptomatic group (Group B)

\begin{tabular}{llll}
\hline & Group A (N=405) & Group B (N=141) & $P$ \\
\hline $\begin{array}{l}\text { Age (y) } \\
\text { Number of }\end{array}$ & $35.09 \pm 5.32$ & $34.00 \pm 4.83$ & $P=0.148$ \\
$\begin{array}{l}\text { C-section } \\
\text { deliveries }\end{array}$ & & & \\
One & $356(87.9 \%)$ & $102(72.3 \%)$ & $P<0.05$ \\
Two & $46(11.4 \%)$ & $36(25.5 \%)$ & \\
More than two & $3(0.7 \%)$ & $3(2.1 \%)$ & \\
Uterus position & & & \\
Anteflexion & $242(59.8 \%)$ & $65(46.1 \%)$ & $P<0.05$ \\
Meso-position & $20(4.9 \%)$ & $6(4.3 \%)$ & \\
Retroflexion & $143(35.3 \%)$ & $70(49.6 \%)$ & \\
Hysteromyoma & $71(17.5 \%)$ & $15(10.6 \%)$ & $P=0.053$ \\
CSD (N) & $95(23.5 \%)$ & $114(80.9 \%)$ & $P<0.05$ \\
CSD parameters & & & \\
Length (mm) & $5.09 \pm 2.31$ & $5.96 \pm 2.64$ & $P<0.05$ \\
Depth (mm) & $6.62 \pm 3.05$ & $8.39 \pm 3.72$ & $P<0.05$ \\
Width (mm) & $8.89 \pm 4.15$ & $11.44 \pm 4.98$ & $P<0.05$ \\
D/W & $1.85 \pm 1.55$ & $3.84 \pm 3.08$ & $P<0.05$ \\
TRM (mm) & $5.39 \pm 3.34$ & $3.22 \pm 2.33$ & $P<0.05$ \\
\hline
\end{tabular}

only had one previous CS in Group A was 356 (87.9\%) versus the $102(72.3 \%)$ in Group B. The uterine positions of two groups of patients on ultrasound examination were compared, anterior position: 242 (59.8\%) vs. 65 (46.1\%); meso-position: 20 (4.9\%) vs. 6 (4.3\%); and retroposition: $143(35.3 \%)$ vs. 70 (49.6\%), $P<0.05$. The detection rates of CSD in the two groups were dramatically different, $23.5 \%$ vs. $80.9 \%, P<0.05$. The length, depth, width and TRM of the CSD were all significantly different between the two groups (Table 2). 


\section{Clinical characteristics of the patients in Group C and Group D}

Group C $(\mathrm{N}=337)$ included women who had no CSDs on transvaginal ultrasound, Group D $(\mathrm{N}=209)$ included women who had detected CSDs on transvaginal ultrasound. The average age of two groups patients was $35.04 \pm 5.41$ (Group C) versus $34.44 \pm 4.88$ (Group D) years old, and the median age of two groups was 34 years old. The number of patients who only had one previous CS in Group C was 297 (88.1\%) versus the 161 (77\%) in Group D, $P<0.05$. The uterine position of two groups on transvaginal ultrasound were compared, anterior position: $215(63.8 \%)$ versus 92 (44\%), meso position: 11 (3.3\%) versus 15 (7.2\%), and retroposition: 111 (32.9\%) versus $108 \%, P<0.05$. The mean TRM measurements in the two groups were $6.54 \pm 2.13$ versus $4.21 \pm 3.03$, $P<0.05$. The TRM in the non-CSD group was significantly thicker than that in the CSD group (Table 3 ).

\section{Receiver operating characteristic curves and logistic analysis}

The clinical symptoms, uterine position, and TRM in the women with CSDs were significantly different from those in the women without CSDs. The results of logistic multivariate regression analysis are shown in Table 4 . The ROC curves of the CSD and non-CSD groups were drawn with these three values (Fig. 2). The curves obtained from the three values together to predict CSD indicated a cutoff value of 0.346 with a sensitivity of $84.6 \%$ and a specificity of $55.9 \%$ (95\% CI $0.76-0.85$ ). In terms of individual indicators to predict CSDs, the TRM with TVS had a high predictive value. The ROC curve indicated that at the cutoff value of $4.15 \mathrm{~mm}$, the TRM variable had a sensitivity of $87.8 \%$ and a specificity of $71.3 \%$ for predicting CSDs (95\% CI 0.723-0.819), and the area under the ROC curve was 0.779 (Table 5).

\section{Discussion}

In China, the proportion of Caesarean sections (CS) performed In 2010 was $35-58 \%$, which has also aroused widespread concern about CSDs [11]. With the increasing CS rate and the implemented two-child policy, the complications of CSDs, such as secondary infertility, prolonged menstrual bleeding, even uterine rupture during a subsequent pregnancy, have emerged as important clinical problems, which serious impact on women's reproductive health [12]. Therefore, it is necessary to evaluate cesarean section scar before the next pregnancy.

CSDs can be detected by transvaginal ultrasound (TVS) [2, 13], sonohysterography (SHG), hysterography, hysteroscopy (HSC), and magnetic resonance imaging (MRI) [14-17]. Among these methods, TVS is a simple,noninvasive and low-cost examination that should be considered as the first choice for screening CSDs [18],

Table 3 Characteristics of clinical data in non-CSD group and CSD group

\begin{tabular}{|c|c|c|c|}
\hline & Group C $(\mathrm{N}=337)$ & Group $D(N=209)$ & $P$ \\
\hline Age (y) & $35.04 \pm 5.41$ & $34.44 \pm 4.88$ & $P=0.355$ \\
\hline Symptomatic & $27(8.0 \%)$ & $114(54.5 \%)$ & $P<0.05$ \\
\hline \multicolumn{4}{|c|}{ Number of C-section deliveries } \\
\hline One & $297(88.1 \%)$ & $161(77 \%)$ & \\
\hline Two & $38(11.3 \%)$ & $44(21.1 \%)$ & $P<0.05$ \\
\hline More than twice & $2(0.6 \%)$ & $4(1.9 \%)$ & \\
\hline \multicolumn{4}{|l|}{ Uterus position } \\
\hline Anteflexion & $215(63.8 \%)$ & $92(44 \%)$ & $P<0.05$ \\
\hline Meso-position & $11(3.3 \%)$ & $15(7.2 \%)$ & \\
\hline Retroflexion & $111(32.9 \%)$ & $102(48.8 \%)$ & \\
\hline Hysteromyoma & $61(18.1 \%)$ & $25(12 \%)$ & $P=0.056$ \\
\hline TRM (mm) & $6.54 \pm 2.13$ & $4.21 \pm 3.03$ & $P<0.05$ \\
\hline
\end{tabular}

Table 4 Logistic regression analysis results

\begin{tabular}{llllllll}
\hline & B & SE & Wald & df & P & Exp (B) & 95\% Cl \\
\hline Symptom & 2.95 & 0.26 & 70.31 & 1 & 0.00 & 8.98 & $5.38-14.99$ \\
Uterus position & 0.29 & 0.11 & 6.68 & 1 & 0.10 & 1.33 & $1.07-1.66$ \\
TRM & 0.25 & 0.04 & 31.05 & 1 & 0.00 & 0.78 & $0.72-0.85$ \\
\hline
\end{tabular}



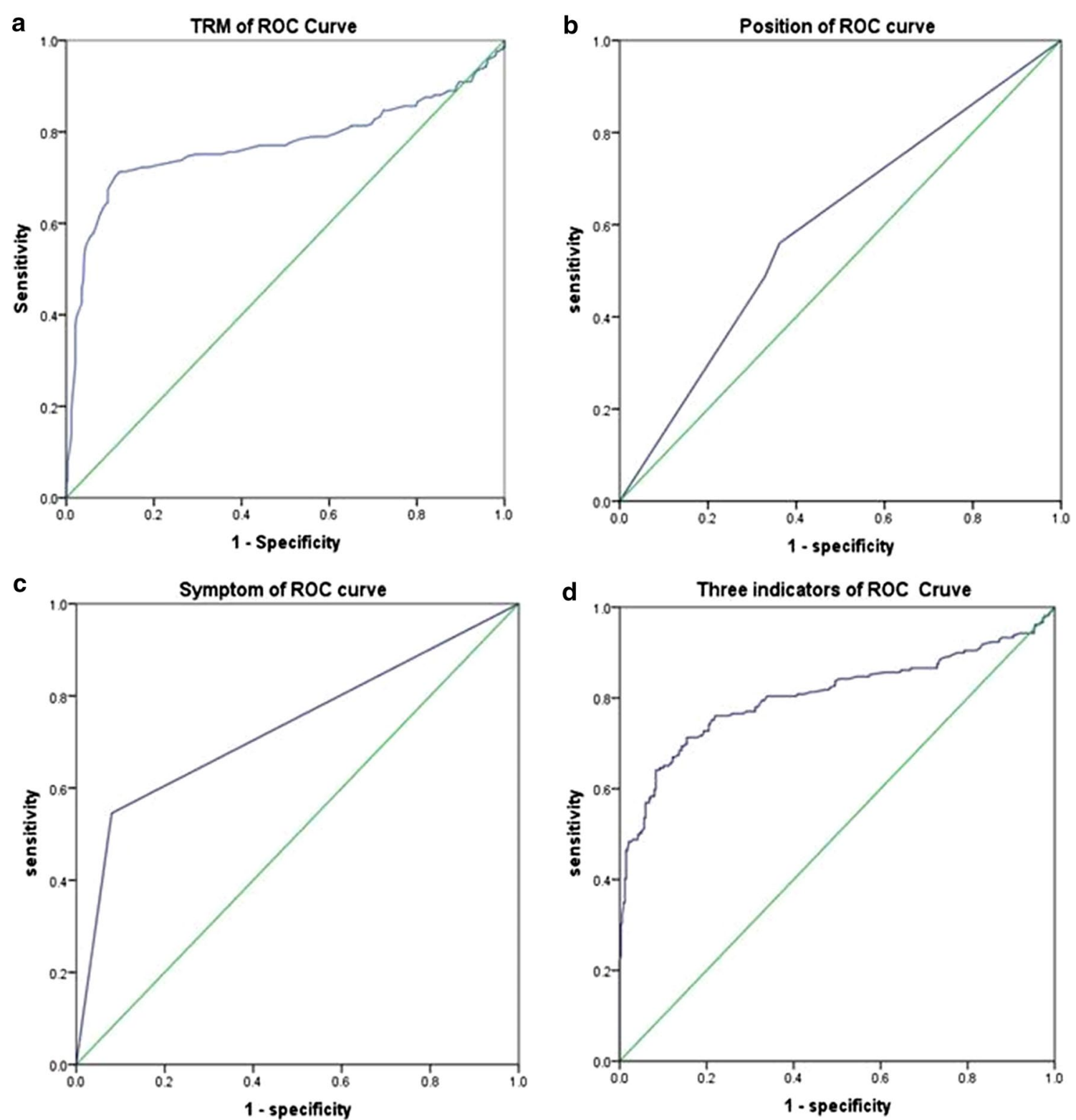

Fig. 2 Receiver operating characteristic curves, a ROC curve of TRM thickness; b ROC curve of uterine position; c ROC curve of symptoms; $\mathbf{d}$ ROC curve of all three indicators

Table 5 Receiver operating characteristic curves

\begin{tabular}{|c|c|c|c|c|c|c|c|}
\hline Indicator & Cut-off & AUC & $P$ & Sensitivity & Specificity & Youden's index & $95 \% \mathrm{Cl}$ \\
\hline Symptom & 0.522 & 0.733 & $P<0.05$ & 0.545 & 0.92 & 0.465 & $0.686-0.779$ \\
\hline $\begin{array}{l}\text { Number of C-section } \\
\text { deliveries }\end{array}$ & 0.444 & 0.556 & $P<0.05$ & 0.23 & 0.881 & 0.111 & $0.506-0.606$ \\
\hline Uterus position & 0.389 & 0.603 & $P<0.05$ & 0.56 & 0.638 & 0.198 & $0.554-0.652$ \\
\hline TRM & 4.15 & 0.771 & $P<0.05$ & 0.878 & 0.713 & 0.591 & $0.723-0.819$ \\
\hline Three indicators & 0.346 & 0.805 & $P<0.05$ & 0.713 & 0.846 & 0.559 & $0.761-0.848$ \\
\hline
\end{tabular}


nevertheless, unskilled gynecologists or the use of a lowresolution ultrasound machine can miss defects during routine ultrasound scans, especially if the operator does not suspect a CSD and there does not look for a defect.

Singhl et al. [19] evaluated scar thickness in pregnant patients with previous caesarean section by TVS and magnetic resonance imaging (MRI) to determine the precision of radiologically measured scar thickness with the actual measured scar thickness. These measurements were correlated with each other and with the scar thickness measured during elective repeat caesarean section using a caliper. The study showed that the thickness measured with TVS had a better correlation coefficient with the actual scar thickness than the thickness measured with MRI ( $R=0.72$ vs. $R=0.59$ ). Marasinghe's research came to a similar conclusions [20]. These authors all relieved that TVS could be considered the preferred modality for antenatal scar thickness measurements.

Therefore, our study established a CSD risk assessment model by applying TVS to evaluate the uterine scar healing of 607 women with a history of cesarean section. The results showed that the TRM measured with TVS effectively predicted CSDs when TRM was less than $4.15 \mathrm{~mm}$, and uterine incision diverticulum was more easily detected below this thickness threshold. In other words, if the detected TRM was less than $4.15 \mathrm{~mm}$ by ultrasonography, but a CSD was not found, it was suggested that the scar condition should be re-evaluated by other imaging examinations. This method could avoid missed diagnoses of poor uterine scar healing. But when the TRM was more than $4.15 \mathrm{~mm}$, less than $5.39 \mathrm{~mm}$, CSD can not be detect by TVS, there may be a small diverticulum and ultrasonic sensitivity cannot be detected, so we think this kind of diverticulum only cause menstrual blood accumulation but does not affect the thickness of the next pregnancy,meanwhile, when the thickness is greater than $5.39 \mathrm{~mm}$, healing can be thought of a good, close to the normal muscle layer thickness. This theory still needs to be further verified by expanding the sample size in the future research.

A study by Hayakawa et al., in turn, the study involved 137 women demonstrated that double-layer interrupted sutures reduced the probability of poor myometrium healing after CS 30-38 days after surgery [21]. Another randomized study that enrolled 78 women with TRM evaluated by TVS after surgery found that suturing all the myometrial layers, including the endometrium, reduced the risk for poor healing and incomplete regeneration [22, 23]. A retrospective study by Sevket et al., which enrolled the longest follow-up period of 6 months after surgery, showed that the use of a double layer locked suture promoted complete healing [24]. Finally, a commentary assay on the evaluation of scar healing after CS points out that several research's have also used 6-9 months after surgery to evaluate scar healing, indicating that 6 months after surgery represents a relatively stable state of scar healing [25]. This finding is in keeping with the follow-up results of our previous study about the transvaginal repair of CSDs, which showed that the wound healing was stable in 6 months after surgery [26]. In this study, women were followed up for more than 6 months after cesarean section.

Now, the clinical guidelines about the treatment of CSDs was remain unclear. Several successful surgical treatments for CSDs have been reported in recent years, including hysteroscopic resection, laparoscopic surgery, laparoscopic and hysteroscopic repair, and vaginal repair. In our previous studies, at 6 months after surgery, 80.3\% of patients (94 of 117) reached $\leq 10$ days of menstruation, 48 patients (63.2\%) had no CSDs, and 11 patients (14.5\%) had a $>70 \%$ reduction in CSD volume; additionally, CSDs still existed in more than $40 \%$ of patients after vaginal repair [27]. As long as the TRM increased and their menstrual symptoms improved, the repair surgery could still be considered effective in increasing the safety of the second pregnancy. However, no clinical guidelines have been issued for the management of CSDs with intermenstrual bleeding and/or thickness of the remaining muscular layer (TRM) or for the residual muscle thickness that is considered the ideal result of a repair. Therefore, we need to evaluate uterine scar healing in women after cesarean section to obtain the average level of scar recovery.

\section{Conclusions}

This study showed that for patients with no clinical symptoms, the mean thickness of the TRM with transvaginal ultrasonography is $5.39 \pm 3.34 \mathrm{~mm}$; for the patients who have clinical symptoms, the mean thickness of the TRM with transvaginal ultrasonography is $3.22 \pm 2.33 \mathrm{~mm}$. We believe that a residual muscle thickness of $5.39 \pm 3.34 \mathrm{~mm}$ could be used as a good reference to predict the recovery of patients with CSDs after repair surgery.

\section{Abbreviations \\ CS: Caesarean section; CSD: Caesarean scar diverticula (defect); TRM: Thickness of the remaining muscular layer; TVU: Transvaginal ultrasound; GDM: Gesta- tional diabetes mellitus; LUS: Lower uterine segment; ROC: Receiver operating characteristic; MRI: Magnetic resonance imaging.}

\section{Acknowledgements}

We thank all of the patients, doctors and nurses who participated in this study.

\section{Authors' contributions}

XZ: Manuscript writing, data analysis and manuscript revision. HQ: Data collection and interpretation of data TZ: have drafted the work and substantively revised it. YZ: Manuscript revision, design of the work. XW: design of the work, manuscript editing. All authors read and approved the final manuscript. 


\section{Funding}

This study was supported by a Key Grant from the Shanghai Scientific and Technology Commission (15411961600), National Key R\&D Program of China (2020YFC2002800) and an action plan from Shenkang (16CR4028A). Key Grant from the Shanghai Scientific and Technology Commission (15411961600): design of the study, collection, analysis. National Key R\&D Program of China (2020YFC2002800) and Action plan from Shenkang (16CR4028A): interpretation of data, and in writing the manuscript.

\section{Availability of data and materials}

The datasets generated and/or analyzed during the current study are not publicly available due to potential for individual and organizational privacy to be compromised. Reasonable requests for parts of the data will be considered by the corresponding author. The Shanghai First Maternity and Infant Hospital, Tongji University granted these permissions.

\section{Declarations}

\section{Ethics approval and consent to participate}

All study procedures were approved by the Ethics Committee of Shanghai First Maternity and Infant Hospital, affiliated with Tongji University (KS1512). This ethics was obtained by corresponding author, Xipeng Wang. We conducted research using identifiable electronic medical record data. The research project did not involve personal privacy and commercial interests and was no longer accessible to participants. Therefore, the ethics committee approved the waiver of signed written informed consent and approved the procedure for verbal consent. At the same time, after obtaining ethical approval, we tried to contact the participants before using the data and notified them by phone. A patient who couldn't be reached by phone, whose data could be used directly. For participants with missing follow-up data, oral informed consent was obtained before the start of each telephone interview. All processes have been recorded and saved. Administrative permissions were required to access and use the medical records described in our study.

\section{Consent to publish}

Not applicable.

\section{Competing interests}

The authors declare that they have no competing interests.

\section{Author details}

${ }^{1}$ Department of Obstetrics and Gynecology, Xinhua Hospital, Affiliated to Shanghai Jiaotong University, No. 1665 Kong Jiang Road, Shanghai 200092, China. ${ }^{2}$ Department of Gynecology, Qingdao Municipal Hospital, Shandong 266071, China. ${ }^{3}$ Department of Ultrasound, Shanghai First Maternity and Infant Hospital, Affiliated To Tongji University, Shanghai, China.

\section{Received: 9 November 2020 Accepted: 30 April 2021}

Published online: 13 May 2021

\section{References}

1. Pomorski M, Fuchs T, Zimmer M. Prediction of uterine dehiscence using ultrasonographic parameters of Cesarean section scar in the nonpregnant uterus: a prospective observational study. BMC Pregnancy Childbirth. 2014;14:365.

2. Jordans I, de Leeuw RA, Stegwee SI. Sonographic examination of uterine niche in non-pregnant women: a modified Delphi procedure. Ultrasound Obstet Gynecol. 2019;53(1):107-15.

3. Bij de Vaate AJ, van der Voet LF, Naji O, Witmer M, Veersema S, Brolmann HA, et al. Prevalence, potential risk factors for development and symptoms related to the presence of uterine niches following Cesarean section: systematic review. Ultrasound Obstet Gynecol. 2014;43(4):372-82.

4. Monteagudo A, Carreno C, Timor-Tritsch IE. Saline infusion sonohysterography in nonpregnant women with previous cesarean delivery: the "niche" in the scar. J Ultrasound Med. 2001;20(10):1105-15.

5. Vitale SG, Ludwin A, Vilos GA, et al. From hysteroscopy to laparoendoscopic surgery: what is the best surgical approach for symptomatic isthmocele? A systematic review and meta-analysis. Arch Gynecol Obstet. 2020;301(1):33-52.

6. Van der Voet LF, Bij de Vaate AM, Veersema S, Brolmann HA, Huirne JA. Long-term complications of caesarean section: The niche in the scar: a prospective cohort study on niche prevalence and its relation to abnormal uterine bleeding. BJOG. 2014;121(2):236-44.

7. Gulino FA, Pappalardo E, Ettore C, Laganà AS, Capriglione S, Ettore G. Caesarean scar pregnancy: descriptive paper of three different types of management on a series of clinical cases. Menopause. 2020;19(2):61-5.

8. Zhou D, Wu F, Zhang Q. Clinical outcomes of hysteroscopy-assisted transvaginal repair of cesarean scar defect. J Obstet Gynaecol Res. 2020;46(2):279-85.

9. Kok N, Wiersma IC, Opmeer BC, de Graaf IM, Mol BW, Pajkrt E. Sonographic measurement of lower uterine segment thickness to predict uterine rupture during a trial of labor in women with previous Cesarean section: a meta-analysis. Ultrasound Obstet Gynecol. 2013;42(2):132-9.

10. Bergeron ME, Jastrow N, Brassard N, Paris G, Bujold E. Sonography of lower uterine segment thickness and prediction of uterine rupture Obstet Gynecol. 2009;113(2):520-2.

11. Donnez O, Donnez J, Orellana R, Dolmans MM. Gynecological and obstetrical outcomes after laparoscopic repair of a Cesarean scar defect in a series of 38 women. Fertil Steril. 2017;107(1):289-96.

12. Van der Voet LF, Vervoort AJ, Veersema S, BijdeVaate AJ, Brolmann HA, Huirne JA. Minimally invasive therapy for gynaecological symptoms related to a niche in the caesarean scar: a systematic review. BJOG. 2014;121(2):145-56.

13. Spong CY, Queenan JT. Uterine scar assessment: how should it be done before trial of labor after Cesarean delivery? Obstet Gynecol. 2011;117(3):521-2

14. Menada Valenzano M, Lijoi D, Mistrangelo E, Costantini S, Ragni N. Vaginal ultrasonographic and hysterosonographic evaluation of the low transverse incision after caesarean section: correlation with gynaecological symptoms. Gynecol Obstet Investig. 2006;61(4):216-22.

15. Regnard C, Nosbusch M, Fellemans C, Benali N, van Rysselberghe $M$, Barlow $\mathrm{P}$, et al. Caesarean section scar evaluation by saline contrast sonohysterography. Ultrasound Obstet Gynecol. 2004;23(3):289-92.

16. Lal K, Tsomo P. Comparative study of single layer and conventional closure of uterine incision in cesarean section. Int J Gynaecol Obstet. 1988;27(3):349-52.

17. Osser OV, Jokubkiene L, Valentin L. High prevalence of defects in cesarean section scars at transvaginal ultrasound examination. Ultrasound Obstet Gynecol. 2009;34(1):90-7.

18. Fabres C, Aviles G, De La Jara C, Escalona J, Munoz JF, Mackenna A. The cesarean delivery scar pouch: clinical implications and diagnostic correlation between transvaginal sonography and hysteroscopy. J Ultrasound Med. 2003;22(7):695-700.

19. Singh N, Tripathi R, Mala YM, Dixit R, Tyagi S, Batra A. Comparison of scar thickness measurements using trans-vaginal sonography and MRI in cases of pregnancy with previous caesarean section. Do they correlate with actual scar thickness? J Obstet Gynaecol. 2013;33(8):810-3.

20. Marasinghe JP, Senanayake H, Randeniya C, Seneviratne HR, Arambepola C, Devlieger R. Comparison of transabdominal versus transvaginal ultrasound to measure thickness of the lower uterine segment at term. Int J Gynaecol Obstet. 2009;107(2):140-2.

21. Hayakawa H, Itakura A, Mitsui T, Okada M, Suzuki M, Tamakoshi K, et al. Methods for myometrium closure and other factors impacting effects on cesarean section scars of the uterine segment detected by the ultrasonography. Acta Obstet Gynecol Scand. 2006;85(4):429-34.

22. Yazicioglu F, Gokdogan A, Kelekci S, Aygun M, Savan K. Incomplete healing of the uterine incision after caesarean section: Is it preventable? Eur J Obstet Gynecol Reprod Biol. 2006;124(1):32-6.

23. Vikhareva Osser $O$, Valentin L. Risk factors for incomplete healing of the uterine incision after caesarean section. BJOG Int J Obstet Gynaecol. 2010;117(9):1119-26.

24. Sevket O, Ates S, Molla T, Ozkal F, Uysal O, Dansuk R. Hydrosonographic assessment of the effects of 2 different suturing techniques on healing of the uterine scar after cesarean delivery. Int J Gynaecol Obstet. 2014;125(3):219-22.

25. Laganà AS, Cromi A, Tozzi R, Franchi M, Lukanović D, Ghezzi F. Uterine scar healing after cesarean section: managing an old surgery in an evidencebased environment. J Investig Surg. 2018;32:1521-53. 
26. Zhou X, Yao M, Zhou J. Defect width: the prognostic index for vaginal repair of cesarean section diverticula. Arch Gynecol Obstet. 2017;295(3):623-30.

27. Zhou J, Yao M, Wang H, Tan W, Chen P, Wang X. Vaginal repair of cesarean section scar diverticula that resulted in improved postoperative menstruation. J Minim Invasive Gynecol. 2016;23:969-78.

\section{Publisher's Note}

Springer Nature remains neutral with regard to jurisdictional claims in published maps and institutional affiliations.
Ready to submit your research? Choose BMC and benefit from:

- fast, convenient online submission

- thorough peer review by experienced researchers in your field

- rapid publication on acceptance

- support for research data, including large and complex data types

- gold Open Access which fosters wider collaboration and increased citations

- maximum visibility for your research: over $100 \mathrm{M}$ website views per year

At BMC, research is always in progress.

Learn more biomedcentral.com/submissions 